\title{
气浮悬吊式太阳翼重力补偿装置的设计与验证 ${ }^{*}$
}

\author{
吴跃民 ${ }^{1}$ 罗 强 $^{1}$ 王 晛 ${ }^{1}$ 刘 渘 $^{1}$ 孙建辉 $^{2}$ \\ (1. 中国空间技术研究院总体部 北京 100094; \\ 2. 浙江工业大学机械工程学院 杭州 310032)
}

\begin{abstract}
摘要: 针对滚轮滑车悬吊式重力补偿装置运行阻力大、气浮支撑式补偿装置无法满足机构向气浮平台方向运动的问题, 提出 了基于龙门架式支撑结构与横、纵双向气浮导轨相结合的悬吊式重力补偿装置设计方案。采用多段拼接技术解决了超长纵向导 轨生产与装调难题; 采用在纵向导轨拼缝两侧增设节流孔的方法, 提升了纵向滑车在拼缝处的运行平稳性; 在纵向导轨上采 用倒 “ $\mathrm{V}$ ” 形工作表面, 解决了因外力未通过质心可能引起横向导轨运行偏转的问题; 采用挤压成型中空铝型材与碳纤维增 强梁相结合的方法实现了大跨距横向导轨的减重与抗弯刚度的提升。经分析与试验验证, 在单个悬吊点处悬挂 $90 \mathrm{~kg}$ 负载, 该装置最大运行阻力系数不超过 $0.037 \%$, 运行范围可达 $2 \mathrm{~m} \times 20 \mathrm{~m}$, 在速度不超过 $1 \mathrm{~m} / \mathrm{s}$, 加速度不超过 $0.3 \mathrm{~m} / \mathrm{s}^{2}$ 的使用条件下, 具有良好的动态跟随能力, 可满足太阳翼、天线等大型空间可展开机构地面展开试验与性能测试的需求。
\end{abstract}

关键词: 气浮滑车; 重力补偿; 悬吊系统; 可展开机构; 地面展开试验

中图分类号: V416

\section{Design and Verification of Air-floating Suspension Gravity Compensation Device for Solar Wing}

\author{
WU Yuemin ${ }^{1} \quad$ LUO Qiang $^{1} \quad$ WANG Xian $^{1} \quad$ LIU Ying $^{1} \quad$ SUN Jianhui ${ }^{2}$
}

(1. Institute of Spacecraft System Engineering, China Academy of Space Technology, Beijing 100094; 2. Institute of Mechanical Engineering, Zhejiang University of Technology, Hangzhou 310032)

\begin{abstract}
Aiming at the problem that the running resistance of the wheeled trolley suspension gravity compensation device is large and the air-floating support compensation device cannot meet the requirement of the mechanism deploy against the air-floating platform, a suspension gravity compensation device composed of the gantry-type support structure and the transverse and longitudinal two-way air-floating guides is proposed. The multi-segment splicing technology is used to solve the problem of production and adjustment of super long longitudinal guide rails; the method of adding throttle holes on both sides of the longitudinal guide joints is adopted to improve the running stability of the longitudinal air-floating trolley at the joints; The inverted "V" shaped working surface solves the problem of transverse rail running deflection caused by the external force not passing through the centroid; the combination of the extruded hollow aluminum profile and the carbon fiber reinforced beam realizes the weight reduction and bending stiffness increase of the large span transverse guide rail. After analysis and test verification, in case of hanging a $90 \mathrm{~kg}$ load at a single suspension point, the maximum running resistance coefficient of the device is not more than $0.037 \%$, the operating range is up to 2 $\mathrm{m} \times 20 \mathrm{~m}$. In case of a follow speed not more than $1 \mathrm{~m} / \mathrm{s}$ and the acceleration is less than $0.3 \mathrm{~m} / \mathrm{s}^{2}$, the device has good dynamic following ability, which can meet the requirements of ground deployment test and performance test of large space deployable mechanisms such as solar arrays and antennas.
\end{abstract}

Key words: air-floating trolley; gravity compensation; suspension system; deployable mechanism; ground deployment test

\section{0 前言}

重力补偿装置在太阳翼、天线、机械臂等复杂 空间可展开机构研制中应用非常广泛 ${ }^{[1-4]}$ 。随着航

* 国家重大科技专项资助项目。20191015 收到初稿, 20200520 收到修 改稿
天技术的发展, 空间可展开机构的规模及活动部件 的重量越来越大, 利用传统的基于滚轮滑车技术的 悬吊式重力补偿设备进行展开试验时 ${ }^{[5-6]}$, 重力补 偿装置引入的附加阻力较大, 可能接近甚至达到与 机构展开驱动力相当的水平, 这将导致机构展开运 动失真甚至出现展开失败的情况 ${ }^{[7-9]}$ 。因此, 开展 重载条件下低运行阻力的重力补偿技术研究具有 
重要意义。

针对活动部件质量、质心运动范围均较大的展 开试验需求, 国内外大多采用气浮平台与多组支撑 式气足相结合的气浮支撑式重力补偿装置实现对 活动部件的重力补偿 ${ }^{[10-11]}$ 。该方法利用高压气体流 经节流孔产生的节流效应在气足与气浮平台之间 形成具有一定承载力的气膜, 从而使气足与气浮平 台相互隔离, 达到有效降低气足运行阻力的需求。 在良好气浮条件下, 气足运行阻力系数可降低至 $10^{-5}$ 左右 ${ }^{[12]}$, 可满足低阻力系数需求, 但所需的大 型气浮平台的搭建及在厂房内的移动较为困难。对 于文献[13]所述的在水平、坚直方向均有展开运动 的二维展开型太阳翼来说, 利用气浮平台实施一维 展开后, 将无法再进行坚直方向的展开运动。

为解决气浮支撑式方案存在的问题, 满足大型 空间展开机构在进行地面展开试验时的运动空间需 求, 可采用在龙门架式支撑结构下方铺设专用导轨, 在导轨上运行气浮滑车的气浮悬吊式重力补偿方 案。荷兰Fokker Space 公司早在 1991 年就开展了类 似的应用研究 ${ }^{[14]}$ 。其利用气浮轴承对传统的滚轮滑 车悬吊式重力补偿设备进行改造, 实现了悬吊点在 横、纵两个方向的低阻力 (纵向阻力系数为 $1 \%$, 横 向为 $0.1 \%$ )运行。但是, Fokker Space 改造后的重力 补偿装置中单悬吊点的额定承载仅为 $100 \mathrm{~N}$, 悬吊 点的运行范围也比较有限。此后, 国内外相关机构 也开展了类似的应用研究工作 ${ }^{[15-17]}$, 但尚未见到大 运动范围、重载型气浮悬吊式重力补偿装置的研究 报道。

基于对国内外气浮技术与悬吊式重力补偿装置 的设计现状和存在问题的分析, 结合国内某大型太 阳翼地面展开试验需求, 本文采用龙门架式支撑结 构与横、纵两个方向的全气浮导轨相结合的技术方 案, 研制了一套悬吊点可在 $2 \mathrm{~m} \times 20 \mathrm{~m}$ 平面范围实 现低阻力运行的重载型气浮悬吊式重力补偿装置, 开展了相关性能测试, 并利用太阳翼、卫星天线等 产品进行了应用验证。

\section{1 需求分析与系统设计}

\section{1 需求分析}

某大型可展开式太阳翼在进行水平方向的展开 试验时, 需要对 4 组太阳电池板分别采取悬吊式重 力补偿措施, 各悬吊点需要满足的技术指标如下: (1) 单个悬吊点承载能力 $\geqslant 90 \mathrm{~kg}$; (2) 相邻悬吊点最 小间距 $\leqslant 35 \mathrm{~mm}$; (3) 悬吊点运动阻力系数 $\leqslant 0.06 \%$; (4) 悬吊点横向运行范围 $\geqslant 2 \mathrm{~m}$; (5) 悬吊点纵向运行
范围 $\geqslant 20 \mathrm{~m}$; 6) 悬吊点最大运行速度 $1 \mathrm{~m} / \mathrm{s}$, 最大 加速度 $0.3 \mathrm{~m} / \mathrm{s}^{2}$ 。

与常规重力补偿装置可实现的性能指标相比, 该需求在悬吊点运行阻力系数、承载能力等方面均 提出了新的挑战。传统的滚轮滑车系统运行阻力系 数通常在 $0.4 \%$ 左右, 无法满足上述需求。气浮滑车 与高精度导轨配合虽可达到更小的阻力系数, 但重 载、大范围运行的气浮滑车对导轨工作面的刚度、 工作状态水平度等提出了较高的要求。如果导轨在 使用过程中发生变形, 或装配过程中水平度调整不 到位, 使气浮滑车在水平度为 $0.3 \mathrm{~mm} / \mathrm{m}$ 的斜坡上 工作时, 由于重力分量引入的运行阻力系数将达到 $0.03 \%$, 叠加其他因素引起的运行阻力后, 将无法 满足运动阻力系数 $\leqslant 0.06 \%$ 的指标要求。另外, 当 供气压力确定后, 为实现大承载需求, 气浮滑车工 作面的面积难以压缩，不超过 $35 \mathrm{~mm}$ 的吊点间距指 标实现难度较大。

\section{2 系统方案设计}

根据需求分析结果, 选择了图 1 所示的总体构 型方案。整个重力补偿装置包括支撑结构、纵向气 浮组件、横向气浮组件、悬吊装置、供气组件(图 1 未示出)等 5 大部分。

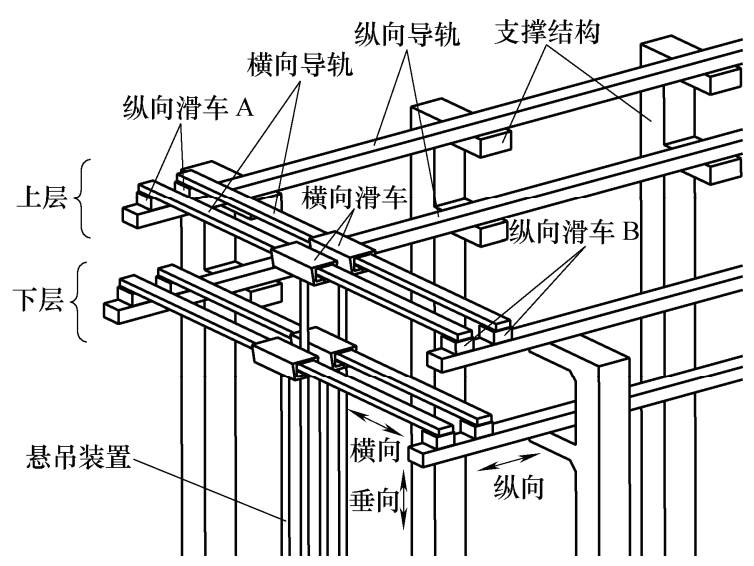

图 1 总体构型方案

支撑结构用于提供纵向气浮组件的安装接口， 具有足够的刚度、强度以及稳定性。纵向气浮组件 包括纵向导轨、纵向滑车(分为 A、B 两种)以及相关 供气管路等，主要用于提供横向导轨的安装接口， 使横向导轨具备沿纵向滑行的能力。横向气浮组件 包括横向导轨、横向滑车以及相关供气管路等，主 要用于实现悬吊装置的安装以及沿横向滑行的能 力。悬吊装置两端分别与横向滑车、太阳翼产品(图 1 未示出)相连。为尽可能减小相邻悬吊点间的最小 距离, 采用了上、下两层导轨错位布局的方案。供 气组件主要用于提供干燥、无油、压力稳定的气源, 以实现对纵向滑车、横向滑车的气浮支撑。 
所有横向气浮组件均采用滑车侧供气方案, 轻 质柔性供气管路从太阳电池板上沿悬吊装置向上铺 设, 直至横向滑车处。纵向气浮组件采用滑车侧与 导轨侧两套供气方案, 在大部分运行区域内仅依靠 滑车侧供气即可, 在局部特殊区域两种供气方案协 同作用, 确保纵向滑车的平稳运行。纵向气浮组件 的供气也采用轻质柔性管路实现, 气路从地面沿支 撑结构爬升至纵向导轨处, 然后分成多个支路, 分 别通向各纵向滑车与纵向导轨。供气组件的组成及 连接关系如图 2 所示。
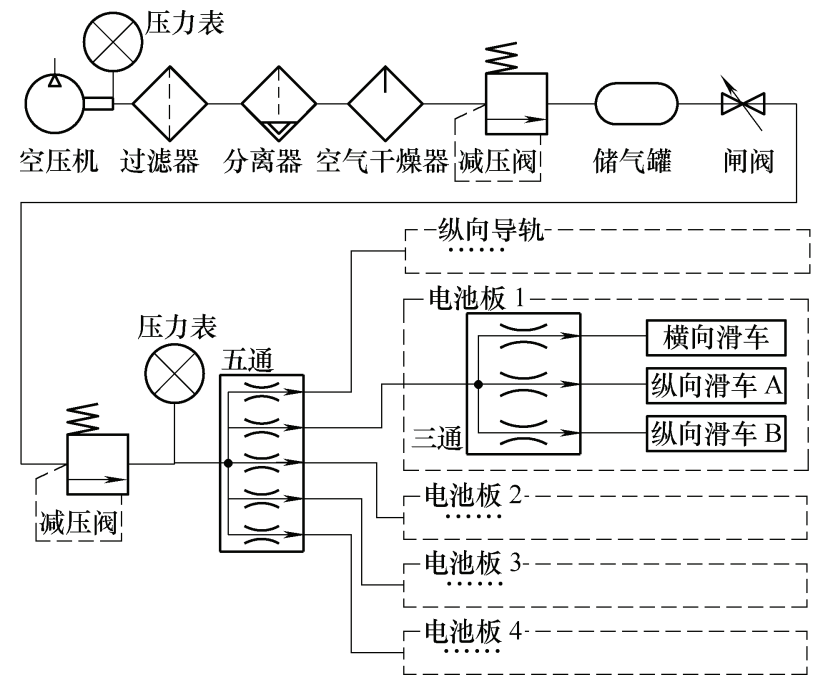

图 2 供气组件的组成及连接关系

\section{2 组件的设计与实现}

\section{1 支撑结构}

为满足太阳翼展开空间需求, 重力补偿装置所 用支撑结构选用龙门架式构型方案, 主要由立柱、 增稳片架以及高刚度上层模块组成, 如图 3 所示。 考虑到厂房地基承载能力有限, 所有结构件均采用 轻质铝合金材料制备, 采用焊接或螺接进行组装, 在立柱根部, 还设计了专用力分散底盘。

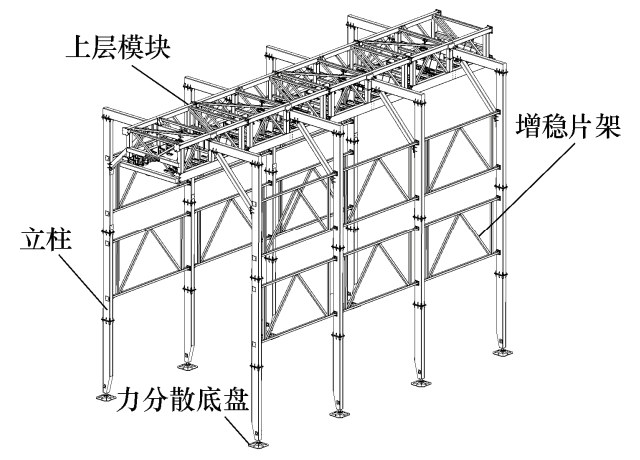

图 3 支撑结构

\section{2 纵向气浮组件}

纵向气浮组件中, 超长导轨的研制是一大难点。
考虑到生产、运输、装调的可实现性, 选择了挤压 成型的中空轻质铝合金型材分段拼接、现场组装的 制备方案, 并在支撑结构上密布支撑点, 以降低对 纵向导轨整体刚度、强度的要求, 尽可能减轻单段 导轨的重量, 降低装调操作难度。

纵向导轨的截面设计为 “伞” 字型, 如图 4 所 示。导轨上侧的倒 “ $\mathrm{V}$ ” 型斜面是工作表面, 与纵 向滑车(图 5)底部对应斜面相互配合。在自身重力以 及横向导轨的作用下, 双斜面设计可有效提升纵向 滑车抗偏转能力, 避免出现两滑车运行不同步的现 象。纵向导轨中部通气孔既是减重孔又是导轨侧供 气管路的组成部分, 同时, 较大的容腔还可起到减 缓供气压力波动的效果。在每段纵向导轨端部, 将 通气孔封闭。利用外部导气管连接相邻导轨之间的 导气孔, 实现气路连通, 如图 6 所示。纵向导轨的 拼接必然会引入㖓隙以及局部台阶, 这对纵向滑车 的平稳运行产生了较大的影响。为此, 在拼缝处设 计了多个节流孔(图 4), 以 “协助” 纵向滑车顺利通 过导轨拼缝。

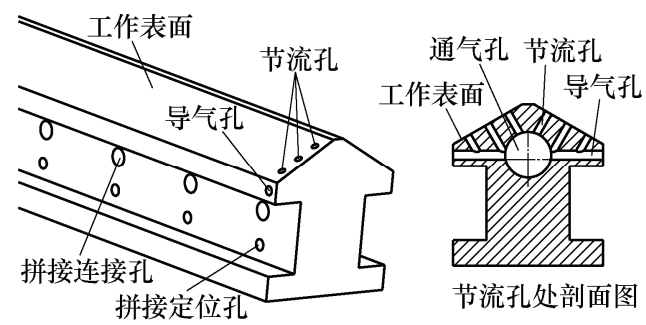

图 4 纵向导轨

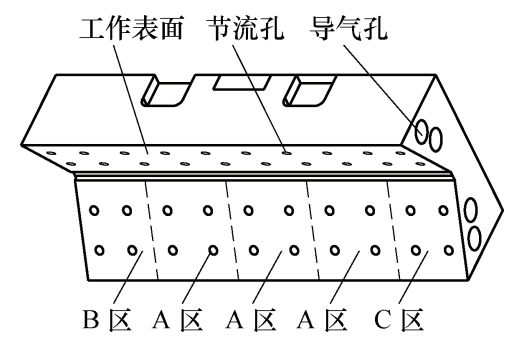

图 5 纵向滑车

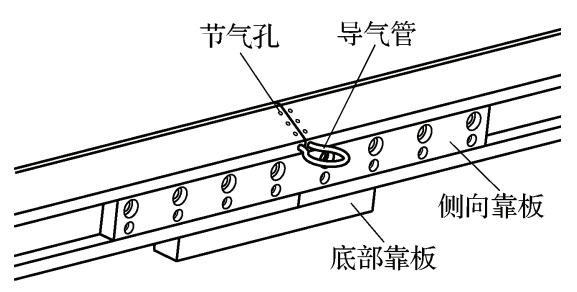

图 6 纵向导轨的拼接

文献[12]指出, 平面气浮系统中气浮面微观平 整度应不超过气膜厚度的 $50 \%$ 。考虑生产工艺可实 现性与成本等因素后, 将纵向导轨工作面粗糙度指 标、多段导轨拼缝处重复定位精度指标均设定为 $5 \mu \mathrm{m}$, 与之配套的滑车气浮面大小、节流孔状态等 均以此为基础进行设计。为防止纵向导轨在加工及 
使用过程中发生形变, 在原材料挤压成型后, 进行 多次应力消除处理。为提升纵向导轨工作表面的耐 磨性, 在导轨完成粗加工后实施表面硬质氧化处理, 最后再采用研磨 ${ }^{[18]}$ 等措施提升光洁度。

超长纵向导轨在支撑结构上的精密装调难度较 大。虽然在每个支撑点处均采用了 3 自由度调节组 件, 但纵导轨的直线度误差、配对使用的纵导轨之 间的平行度误差仍难以消除, 与横导轨连接的两个 纵向滑车之间的间距会随着运行位置的不同而发生 一定的变化。为此, 将纵向滑车设计为 $\mathrm{A} 、 \mathrm{~B}$ 两种 状态, 纵向滑车 A 与横向导轨之间采用铰接设计, 纵向滑车 $\mathrm{B}$ 与横向导轨之间则采用滑动副进行连 接。除此之外, 两种滑车在尺寸规格、气浮工作面 的状态、供气方案等方面完全相同。

\section{3 横向气浮组件}

横向导轨的设计是横向气浮组件研制中的一大 难点。为保证横向滑车在重载条件下仍能满足低阻 力、大行程的运行要求, 横向导轨必须具备足够的 弯曲刚度, 以避免在受载后因变形过大而使横向滑 车运行阻力中重力分量显著增加。另一方面, 在悬 吊式重力补偿装置中, 横向导轨是跟随太阳翼运动 的质量最大的组件, 其跟随运动的动能均来自太阳 翼展开机构。为减小对太阳翼展开运动的影响, 提 升跟随响应速度, 横向导轨的设计还必须满足轻量 化的条件。

碳纤维增强复合材料具有密度小、模量高、稳 定性好等优点, 但单独作为横向导轨使用时, 高精 度工作表面加工难度较大, 整体制作成本较高。铝 合金材料成本低廉且加工简便，但比刚度与纤维增 强复合材料相比有较大差距。为尽可能提升刚度、 降低重量, 同时兼顾制造成本, 选择了铝合金结构 与碳纤维加强梁相结合的横向导轨设计方案, 如 图 7 所示。其中, 铝合金结构首先采用挤压成型工 艺加工成中空、大惯性矩的异型截面坏料, 再进行 局部减重、精加工、表面硬化、研磨等处理; 碳纤 维加强梁按最简单的板材结构加工, 预制连接孔; 最后采用胶铆相结合的方法将两者进行连接。

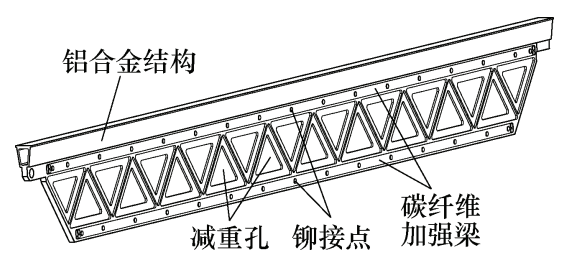

图 7 横向导轨

太阳翼展开过程中, 悬吊点需要沿纵、横两个 方向进行跟随。横向运动时, 跟随运动的主要是悬 吊装置与横向滑车; 纵向运动时, 跟随运动的组件
中还将增加横向导轨与纵向滑车。当悬吊点加速运 行时, 由于惯性作用，横向滑车将会受到纵、横两 个方向的牵引力。为防止在使用过程中发生滑车脱 轨或倾覆, 横向滑车采用了 2 组侧气浮垫与 1 组上 气浮垫相互配合的设计方案, 横向导轨上的 3 个气 浮工作面也按倒置三角形的方式布局, 如图 8 所示。 其中, 侧气浮垫与滑车架之间采用球关节相连, 可 实现与横向导轨侧向气浮工作面的自动匹配。

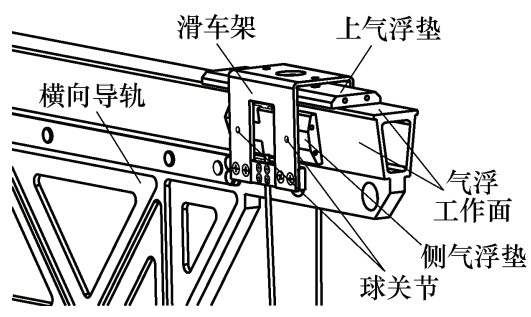

图 8 横向滑车与横向导轨

\section{4 其他组件}

悬吊装置主要由高强度绳索、测力元件、弹性 元件、吊点调节机构等串联连接而成，在满足重载 需求的同时，还可实现悬吊力的实时采集。吊点调 节机构采用轻质铝合金制造，可实现悬吊点在水平 面 $\Phi 20 \mathrm{~mm}$ 范围内任意位置调整与便捷固定。

供气组件包括过滤器、冻干机、减压阀、截止 阀、供气管路以及相关附件等。供气管路中随太阳 翼展开运动发生形变或跟随运动的部分均采用轻质 聚氨酯软管制备; 在穿越太阳翼展开机构处, 供气 管路采用了低阻力旋转接头; 在固定点与活动点之 间, 如纵向滑车与悬吊装置之间, 采用了大 “ $U$ ” 字形布局方案, 以最大限度减小供气管路对太阳翼 展开运动的影响。

\section{3 分析与试验验证}

\section{1 气浮特性}

由于气浮滑车在各自导轨上运行时的速度远低 于工作介质(空气)的流动速度, 因此, 可以将气浮 组件简化为静压空气平面轴承, 并借助相关理论对 其特性进行分析。

气浮滑车与导轨之间的气膜满足控制空气流动 的 Reynolds 方程 ${ }^{[19]}$, 气膜压力分布数学模型可用式 (1)表示

$$
\begin{gathered}
\frac{\partial}{\partial x}\left(h^{3} p \frac{\partial p}{\partial x}\right)+\frac{\partial}{\partial y}\left(h^{3} p \frac{\partial p}{\partial y}\right)= \\
12 \eta \frac{\partial(p h)}{\partial t}+6 \eta\left[v_{x h} \frac{\partial}{\partial x}(p h)+v_{y h} \frac{\partial}{\partial y}(p h)\right]
\end{gathered}
$$

式中, $h$ 为气膜厚度; $p$ 为气膜压力; $\eta$ 为空气动力 
黏度; $v_{x h}$ 为气膜区气体沿 $x$ 方向速度分量在气膜厚 度方向 $z$ 坐标为 $h$ 时的速度边界值; $v_{y h}$ 为 $y$ 方向速 度分量在 $z=h$ 处的速度边界值。

式(1)是一个非线性偏微分方程, 难以求出解析 解, 因此, 在实际应用中采用商用流体力学分析软 件 FLUENT 进行模拟计算。

考虑到纵向气浮滑车设计的对称性, 将图 5 所 示单个工作面用虚线划分为 5 个区域, 选取具有代 表性的 A 区、B 区分别进行建模与分析。分析模型 采用四边形结构网格划分方案, 在节流孔出口附近 采用较为致密的网格以提高计算精度。计算模型选 择标准 $k-\varepsilon$ 湍流模型, 求解器选用基于压力的求解 器。利用理想气体模拟空气, 设置入口压力为 0.3 $\mathrm{MPa}$, 出口压力为 $0 \mathrm{MPa}$, 压力速度耦合算法设置 为 SIM- PLIC 算法。

分析结果表明, 当气膜厚度为 $10 \mu \mathrm{m}$ 时, 纵向 滑车工作面 A 区范围内气压分布如图 9 所示。

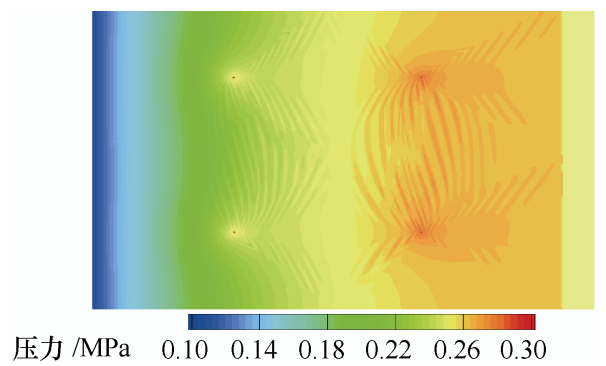

图 9 A 区气压分布

对图 9 所示 $\mathrm{A}$ 区气体压力与面积进行积分, 即 可求出 $\mathrm{A}$ 区所受到的气体支撑力 $F_{A}$ 。同理, 可求出 $\mathrm{B}$ 区、 $\mathrm{C}$ 区所受到的气体支撑力 $F_{B} 、 F_{C}$ 。利用式(2) 即可求出整个纵向滑车在运行过程中所受到的支撑 力 $F_{V}$ 。

$$
F_{V}=2 \times\left(3 F_{A}+F_{B}+F_{C}\right) \cos \theta
$$

式中, $\theta$ 表示纵向滑车工作面与水平面的夹角。

为验证纵向滑车运行过程中气膜厚度与气浮工 作面状态的匹配性, 对滑车承载力与气膜厚度之间 的关系进行了分析, 如图 10 所示。结果表明, 纵向 滑车工作状态下气膜厚度在 $7 \sim 13 \mu \mathrm{m}$ 之间。

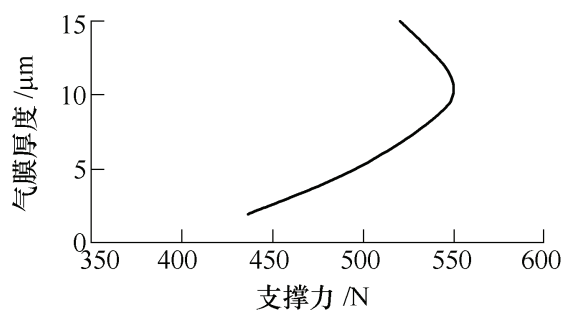

图 10 纵向滑车承载力与气膜厚度的关系

采用同样的方法对横向滑车的气浮特性也进行 了分析验证。

\section{2 导轨刚度}

由前文需求分析可知, 横向或纵向导轨在使用 过程中出现的任何形变, 都将使气浮滑车运行在倾 斜的工作面上, 重力分量将对其运行产生一定的影 响。因此, 在设计阶段, 利用 SolidWorks 完成导轨 建模后，将实体模型导入 MSC/Patran 软件，对横向 导轨、纵向导轨的刚度特性进行了分析。图 11 给出 了横向导轨在中部施加 $900 \mathrm{~N}$ 负载后的变形云图, 从中可以看出, 导轨最大挠度为 $0.1674 \mathrm{~mm}$, 最大 变形转角不超过 $0.012^{\circ}$ 。由于纵向导轨支撑点较为 密集, 在额定载荷作用下变形量较小。

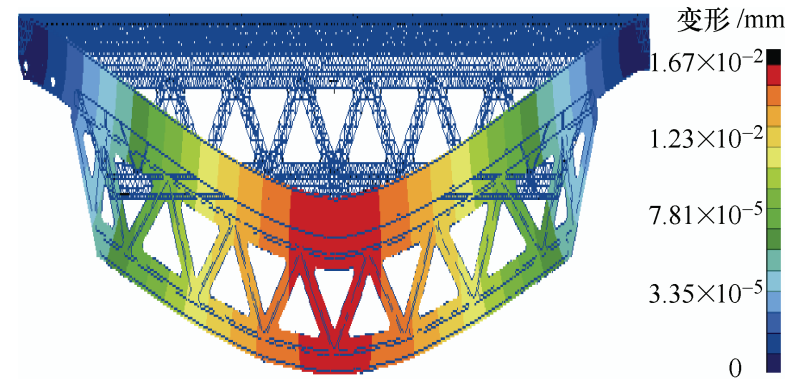

图 11 横向导轨刚度分析结果

在完成导轨组件的加工与装调后, 利用 $90 \mathrm{~kg}$ 配重模拟实际使用工况开展了横向导轨与纵向导轨 的挠曲变形测试, 所得最大变形量为 $0.165 \mathrm{~mm}$, 最 大变形转角为 $0.011^{\circ}$ (均为横向导轨对应参数), 与分 析结果基本一致。

\section{3 滑车运行阻力}

太阳翼在重力补偿装置上进行展开试验时, 纵 向与横向滑车的运行阻力 $F$ 主要包括 2 个部分: (1) 滑车在稳定的气浮状态下匀速运行时受到的气 体摩擦力 $F_{1}$; (2) 导轨装调水平度误差、受载变形 引入的重力分量 $F_{2}$ 。

$F_{1}$ 的精确测定难度很大, 不仅需要用到高精密 的测量设备, 还须消除滑车惯性带来的影响。在实 际工程应用中，通常采用滑车在水平方向的启动推 力 $F_{H}$ 来评估 $F_{1}$ 的大致范围。

$F_{H}$ 的获取可采用图 12 所示方案。首先将支撑 导轨调整为水平状态，然后将滑车、负载等安装到 位, 待整个系统稳定后, 再在滑车一侧施加水平方 向且逐渐增大的推力, 直到滑车开始运动为止, 该 推力的最大值即为启动推力 $F_{H}$ 。由于 $F_{H}$ 非常小, 为防止触碰过程中最大作用力 “淹没” 启动推力, 可采用两个分别安装在滑车与测力计侧面且互斥的 永磁体实现非接触测量(图 12)。为避免因磁场分布 特性引入的系统测量误差，实际应用时，尽可能将 永磁体 $\mathrm{A}$ 与永磁体 $\mathrm{B}$ 相互作用力的方向调整为测力 计测量方向。 


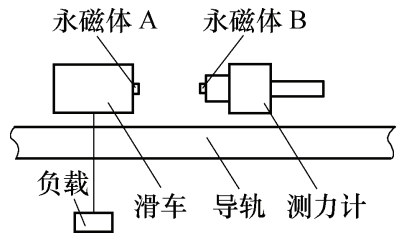

图 12 滑车运行阻力测试方案

测试结果表明, 在 $90 \mathrm{~kg}$ 负载作用下, 纵向滑 车与横向滑车启动推力均不超过 $0.050 \mathrm{~N}$ (测力计精 度为 $\pm 0.005 \mathrm{~N})$, 据此可以认为 $F_{1}$ 最大不超过 $0.055 \mathrm{~N}$ 。按照导轨装调过程中水平度最大偏差为 $0.1 \mathrm{~mm} / \mathrm{m}$, 导轨受载变形产生的最大转角为 $0.012^{\circ}$ 计算, $F_{2}$ 的最大值不超过 $0.273 \mathrm{~N}$ 。将 $F_{1}$ 与 $F_{2}$ 叠加, 折算吊点在 $90 \mathrm{~kg}$ 负载下的运动阻力系数为 $0.037 \%$, 满足不超过 $0.06 \%$ 的指标要求。

\section{4 动态跟随性能验证}

为验证重力补偿装置的动态跟随性能, 在悬吊 点下方悬挂 $90 \mathrm{~kg}$ 重物, 利用特殊的诨引工装在重 物质心处施加水平牵引力, 拖动悬吊点分别沿横向、 纵向进行加速运动, 利用美国 API 公司的 K600 红 外三维测量装置监测悬吊点运行速度及加速度。结 果表明, 在悬吊点运行速度超过 $1 \mathrm{~m} / \mathrm{s}$ 、加速度超过 $0.3 \mathrm{~m} / \mathrm{s}^{2}$ 时, 横向、纵向滑车均能顺畅运行, 不存在 卡滞或其他运行不稳定的现象。

\section{4 应用验证}

整个重力补偿装置装调完成后, 利用某型号真实 太阳翼产品进行了展开试验验证。太阳翼展开过程平 稳有序, 无顿挫、卡滞现象, 展开末了时刻锁定动作 与设计预期一致, 总展开时长约为 $18 \mathrm{~s}$, 连续两次展 开时长差异小于 $1 \mathrm{~s}$, 展开过程照片如图 13 所示。在 太阳翼完全展开并锁定后, 利用该重力补偿装置开展 了整翼展开状态基频测试, 在太阳翼末端给定垂直于 翼面方向的位移激励, 整个翼面显见的摆动周期不少 于 5 个, 对应基频约为 $0.04 \mathrm{~Hz}$, 与仿真分析结果一致。

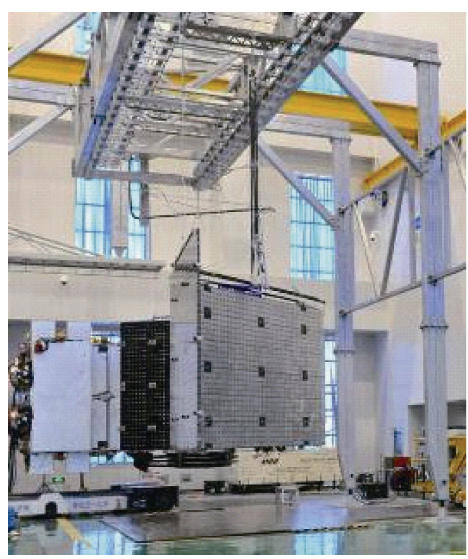

图 13 太阳翼展开试验
除太阳翼外，该装置还成功完成了多套空间可 展开天线的地面展开试验, 并取得了良好的效果。

\section{5 结论}

（1）本文提出的气浮悬吊式重力补偿装置, 实 现了重载条件下悬吊点在 $2 \mathrm{~m} \times 20 \mathrm{~m}$ 范围内低阻力 运行的功能; 与常规的滚轮滑车悬吊式重力补偿装 置相比, 悬吊点运行阻力可降低 1 个数量级以上; 与气浮支撑式重力补偿装置相比, 在提升使用便捷 性的同时，还可满足展开机构在水平、坚直方向均 需展开时的运动空间要求。

(2) 分析及试验验证结果表明, 在 $90 \mathrm{~kg}$ 额定负 载下, 悬吊点最大运行阻力系数不超过 $0.037 \%$, 在 速度不超过 $1 \mathrm{~m} / \mathrm{s}$, 加速度不超过 $0.3 \mathrm{~m} / \mathrm{s}^{2}$ 的使用条 件下, 具有良好的动态跟随能力, 满足太阳翼、可 展开天线等大型空间机构地面展开与性能测试的工 程需求。

(3) 重力补偿装置中高精度超长纵导轨多段拼 接技术、拼缝处滑车过缝技术以及纵向导轨倒 “ $\mathrm{V}$ ” 形工作表面的选用等设计理念与生产措施不但可降 低生产难度，提升整体经济性，对类似气浮系统设 计等也具有一定的参考价值。

\section{参 考 文 献}

[1] BLANC A, CHASSOULIER D, CHAMPANDARD F, et al. Solar array root hinge based on shape memory alloy (SMA) actuator[C]// 15th European Space Mechanisms \& Tribology Symposium-ESMATS, September 25-27, 2013, Noordwijk, the Netherlands. 2013: 1-8.

[2] 刘振, 高海波, 邓宗全, 等. 摇臂转向架式星球车单索 重力补偿 $[\mathrm{J}]$. 机械工程学报，2013，49(7): 113-124.

LIU Zhen, GAO Haibo, DENG Zongquan, et al. Gravity compensation for rocker-bogie rovers through single string tension[J]. Journal of Mechanical Engineering, 2013, 49(7): 113-124.

[3] 杨巧龙, 间泽红, 任守志, 等. 套筒驱动的大型可展收 柔性太阳翼地面展开重力卸载研究 $[\mathrm{J}]$. 载人航天, 2017, 23(4): 536-540, 545.

YANG Qiaolong, YAN Zehong, REN Shouzhi, et al. Study on gravity compensation in ground deployment tests of large retractable flexible solar array driven by telescopic boom[J]. Manned Spaceflight, 2017, 23(4): 536-540, 545 .

[4] 贺云, 张飞龙, 杨明毅, 等. 卫星天线展开臂的随动吊 挂重力补偿系统设计 [J]. 机器人, 2018, 40(3): 377-384, 392.

HE Yun, ZHANG Feilong, YANG Mingyi, et al. Design 
of tracking suspension gravity compensation system for satellite antenna deployable manipulator[J]. Robot, 2018, 40(3): 377-384, 392.

[5] TANiMOTO R L, MOORE A, MACDONALD D R, et al. Model and test validation of gravity offload system $[\mathrm{C}] / /$ 48th AIAA/ASME/ASCE/AHS/ASC Structures, Structural Dynamics, and Materials Conference. April 23-26, 2007, Honolulu, Hawaii. AIAA2007-1790: 1-12.

[6] 从强. 空间机构地面重力补偿设备跟踪研究 [J]. 航天 器环境工程, 2012, 29(1): 92-99.

CONG Qiang. An investigation into gravity compensation equipment for space mechanisms[J]. Spacecraft Environment Engineering，2012，29(1): 92-99.

[7] SHPYAKIN I K, MALYSHENKO A M. Comparative analysis of gravity compensation systems for ground tests of deployable solar arrays[C]// 2015 International Siberian Conference on Control and Communications (SIBCON), May 21-23, 2015, Omsk State Technical University, Russia, Omsk. IEEE, 2015: 1-4.

[8] 任守志, 刘立平. 零重力试验装置对太阳翼展开影响分 析[J]. 航天器工程, 2008, 17(6): 73-78.

REN Shouzhi, LIU Liping. Influence of the zero-gravity test facility on the solar array's deployment test[J]. Spacecraft Engineering, 2008, 17(6): 73-78.

[9] FISCHER A, PELLEGRINO S. Interaction between gravity compensation suspension system and deployable structure[J]. Journal of Spacecraft and Rockets, 2000, 37(1): 93-99.

[10] LI Daming, RAO Wei, HU Chengwei, et al. Overview of the Chinese space station manipulator[C]// AIAA SPACE 2015 Conference and Exposition, August 31-September 2, Pasadena, California. 2015: 1-6.

[11] PENN J, JOHNSON C, LEWIS J, et al. GPM solar array gravity negated deployment testing $[\mathrm{C}] / /$ Proceedings of the 42nd Aerospace Mechanisms Symposium, May 14-16, 2014, NASA Goddard Space Flight Center, 2014: 335-348

[12] RYBUS T, SEWERYN K. Planar air-bearing microgravity simulators: review of applications, existing solutions and design parameters[J]. Acta Astronautica, 2016, 120: 239-259.

[13] DING X, LI X. Design and test analysis of a solar array root hinge drive assembly[J]. Chinese Journal of Mechanical Engineering， 2014， 27(5): 909-918.

[14] AAD P E, HENK J C. Fokker space solar array deployment rigs $[\mathrm{C}] / /$ 33rd Aerospace mechanisms symposium, May 19-21, 1999, Pasadena Conference and Exhibition Center, Pasadena, California. 1999: 405- 418.

[15] 励红峰. 零重力多维展开试验装置研究[D]. 杭州: 浙江 工业大学, 2015

LI Hongfeng. Research of zero gravity multidimensional unfolding test apparatus[D]. Hangzhou : Zhejiang University of Technology, 2015.

[16] Testing sentinel-2's solar wing[EB/OL]. https: //www. esa.int/spaceinvideos/Videos/2015/04/Testing_Sentinel-2_ s_solar_wing, 2015-04-08/2019-01-20.

[17] Orion solar array wing deployment test is a success [EB/OL]. https: //www.nasa.gov/feature/orion-solar-arraywing-deployment-test-is-a-success-0, 2017- 08-07/201901-20.

[18] 宋楠, 姚雪峰, 冯树龙, 等. 高精度双 V 型导轨的研磨 与检测技术 $[\mathrm{J}]$. 长春工业大学学报, 2013，34(2)： 184-189.

SONG Nan, YAO Xuefeng, FENG Shulong, et al. Grind and detection of high-precision double V-type rail [J]. Journal of Changchun University of Technology, 2013, $34(2): 184-189$

[19] 陈改革, 杨涛, 陈立, 等. 静压空气平面轴承特性与节 流器间距关系的仿真研究 [J]. 西南科技大学学报, 2010, 25(4): 62-67, 72.

CHEN Gaige, YANG Tao, CHEN Li, et al. Simulation research on the relationship between orifice spacing and the characteristics of flat pad aerostatic bearing $[\mathrm{J}]$. Journal of Southwest University of Science and Technology, 2010, 25(4): 62-67, 72.

作者简介: 吴跃民, 男, 1980 年出生, 高级工程师。主要研究方向为航 天器结构机构设计与验证。

E-mail: 13520911415@139.com 